\title{
3D MODELLING, ANIMATION AND SIMULATION OF MAMMAL'S MIGRATION ACROSS ROADS
}

\author{
Jozef TALAPKA ${ }^{1, *}$, Ján HLUBÍK ${ }^{2}$, Patrik KAMENCAY ${ }^{3}$, Róbert HUDEC ${ }^{4}$
}

${ }^{1}$ Department of Telecommunications and Multimedia, Faculty of Electrical Engineering, University of Žilina, Univerzitná 8215/1, 01026 Žilina, Slovakia.

2 Department of Telecommunications and Multimedia, Faculty of Electrical Engineering, University of Žilina, Univerzitná 8215/1, 01026 Žilina, Slovakia.

${ }^{3}$ Department of Telecommunications and Multimedia, Faculty of Electrical Engineering, University of Žilina, Univerzitná 8215/1, 01026 Žilina, Slovakia.

4 Department of Telecommunications and Multimedia, Faculty of Electrical Engineering, University of Žilina, Univerzitná 8215/1, 01026 Žilina, Slovakia.

corresponding author: jozef.talapka@fel.uniza.sk.

\begin{abstract}
The presented article is dealing with the new methods which are designated for data collection of mammals migrating across traffic networks. Nowadays, road construction and securing of older roads is usually accompanied by finding new solutions. Because of lack of collected data we have new opportunities how we can collect this input. The article below describes the most efficient method which is suitable for the model creation, process of creation and issues which are connected with the creation of simulations.
\end{abstract}

\section{Keywords:}

3D modelling;

Stereophotogrammetry;

Mammal;

Migration;

Animation.

\section{Introduction}

Due to a growing numbers of road accidents of mammals [1] are needed new graphic simulation methods. A review of literature showed there is no information on the reproducibility and validity of 3D animal photography yet. Earlier studies were performed to investigate the precision of $3 \mathrm{D}$ stereo photogrammetry. The majority of these studies focused on reliably measuring distances between typical anthropometric points on the 3D reconstructed images against corresponding points on live subjects or phantom models (e.g. plaster casts) as a form of validation [2, 3, 4]. Some other studies use more complex methods to obtain and analyse 3D shapes [5, 6].

The outline of this paper is organized as follows. In the Section II, the possibilities of 3D model creation are discussed. The procedure of 3D model creation is listed in section III. The optimization and animation are described in Section IV and Section V respectively. Finally, the Section VII concludes and suggests the future scope.

\section{Options for creation of 3D models}

\subsection{D modelling}

In order to use 3D polygonal modelling, it is necessary to have at least a side view of an object. For the more precise modelling we need more views: front, side, top, bottom, and back. These views cannot be done with living animals; therefore we can use only stuffed objects. In this case, pictures have a perspective what makes modelling distorted. Furthermore, manipulation with stuffed objects is difficult, therefore we are left with only side or sometimes with front and back view modelling. As a consequence, all the models which were bought or created with this method are not as precise as models which were created by photogrammetry or laser scanning. On the Figure 1 is shown a polygonal mesh, texture and final model of bear. 


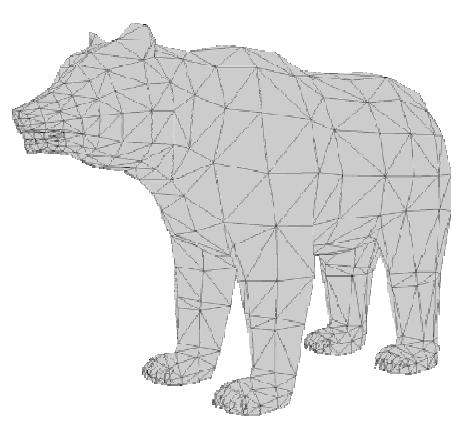

a)

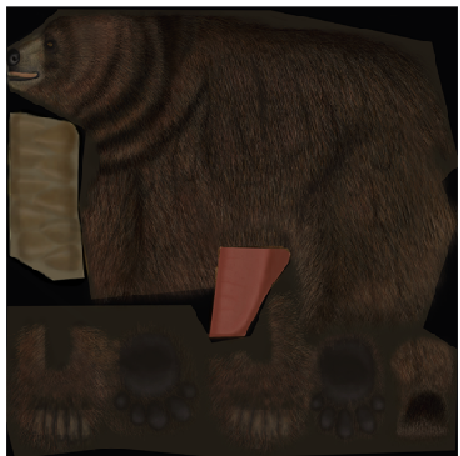

b)

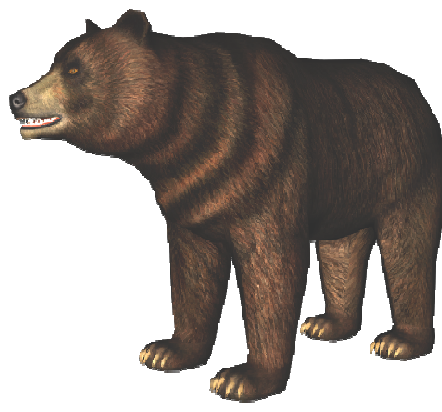

c)

Fig. 1: a) Mesh of model, b) Texture of model, c) Textured mesh.

\subsection{D Laser scanning}

3D scanner (see Fig. 2b) is a device which analyses a real object in order to collect data about its shape and provides complete spatial information about the object. 3D scanner projects a certain light pattern (a cross) on the surface of the object and subsequently examines its deformation. The laser cross on the object is projected via laser. Afterwards, the video camera scans the deformed pattern on the surface of the object. The main advantage of this method is its speed. During the scanning we can place the reflective marks on the scanning pad or directly on the object (see Fig. 2a). In case that both cameras can see at least 3 reflective marks, the system is able to automatically set the position of the object. In order to allow movement it is necessary to see 4 reflective marks. Mutual position of reflective marks is unique and the system is able to connect the previous points with the next scanning.



a)



b)

Fig. 2: a) Pad designed for scanning, b) 3D Laser scanner [8].

The advantage of this method is in its swarm point accuracy which corresponds with real object. The creation of swarm points is relatively quick.

The disadvantage of this method is the adjustment of object's geometry, polygonal optimization the fact that the created model does not contain a texture. The adjustment of scanned polygonal surface is rather time-consuming.

\subsection{Stereo photogrammetry}

When dealing with model's geometry and texture fidelity, stereo photogrammetry serves as the most precise method for 3D model creation. Digital photogrammetry is a modification of analytical photogrammetry, which works with digital pictures. Stereo photogrammetry includes an estimation of three-dimensional point coordinates on the object with the use of measurements which were done in two or more photographic pictures made from various positions (see fig. 3a). Mutual coordinates are identified on each picture. A line of sight (or ray) can be constructed from the camera location to the point on the object. It is the intersection of these rays (triangulation) that determines the threedimensional location of the point. More sophisticated algorithms can exploit other information about the scene that is known. On the Figure $3 b$ is shown placement of cameras around scanned object. 


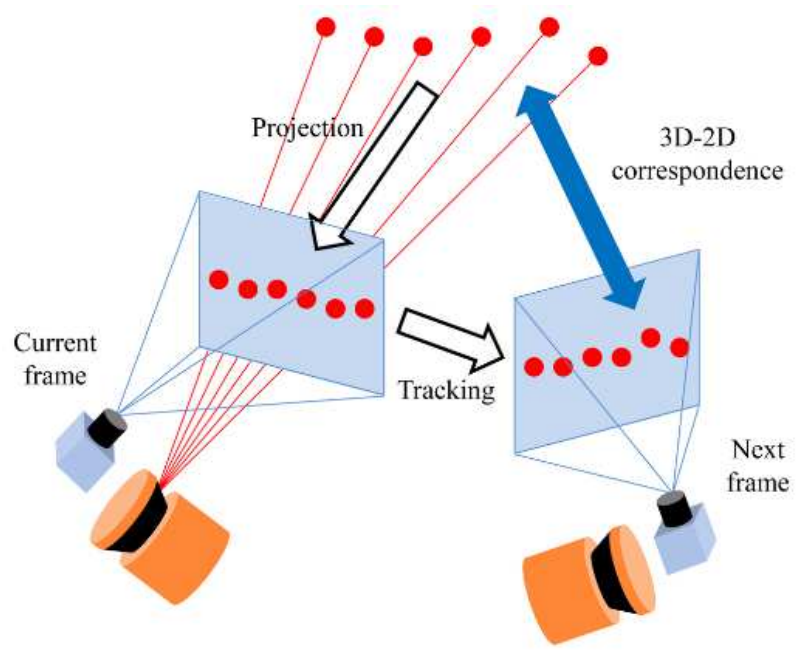

a)

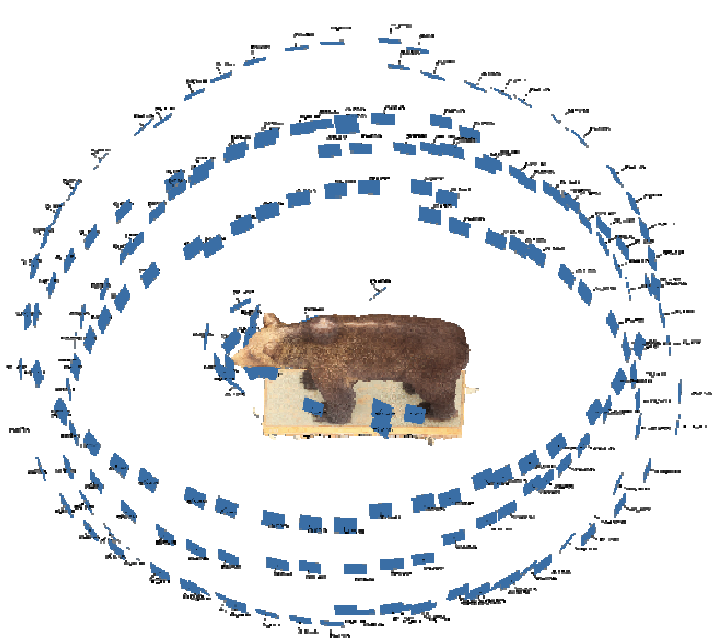

b)

Fig. 3: a) Calculation of 3D scene [9], b) Placement of cameras in the 3D scene.

\subsection{The used method}

During the process (see Fig. 4) we used a combination of photogrammetry and 3D modelling in order to secure the most perfect 3D animal models. With the use of photogrammetry we created 3D models with accurate copy of the texture. These models were subsequently optimized, the amount of polygons was reduced, and the symmetry together with correction of shape was done. The last step was an adjustment of texture for optimized model. After these adjustments the model is ready for animation. We chose this system because of the texture quality, easier and less time-consuming optimization in comparison with 3D modelling from scratch.

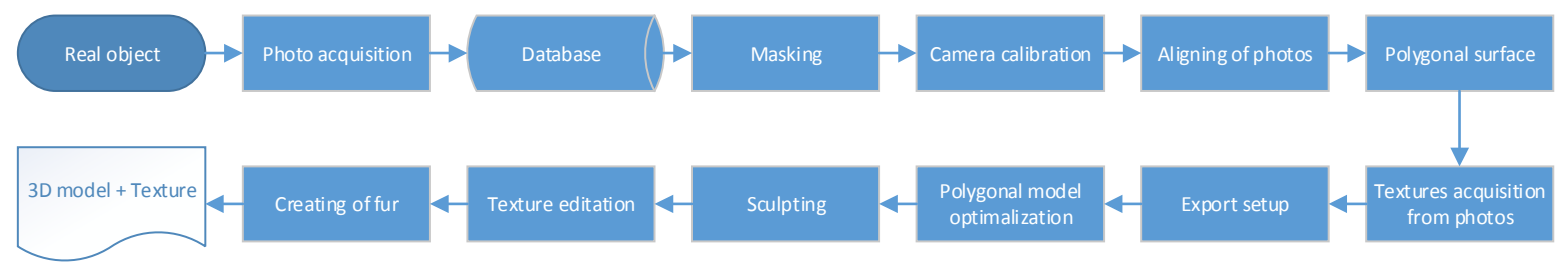

Fig. 4: Flowchart 3D modelling using photogrammetry.

\section{Procedures of 3D creating}

The process involves taking pictures of stuffed animals from various perspectives and sides. In order to get more precise outcome, we took closer pictures of several body parts. Each model contains approximately 150 pictures. These pictures were subsequently adjusted and we also made masks. After that, using these pictures the program created 3D models together with texture in high resolution.

\section{Optimization}

It is necessary to optimize exported models, remove unevenness, and fill holes which emerged after photogrammetric reconstruction. The next important step is to optimize the amount of polygons and to optimize mesh (see Fig. 5). The amount optimization of polygons is necessary because of calculating demands and for simpler manipulation during the animation of the model. The mesh optimization was necessary for correct distribution and density of the grid, especially in the areas around the 3D model's head. The optimization also involves a symmetry correction and the adjustment of position which is necessary for animating - the model must be turned correctly. Because of these adjustments, it is necessary to re-map the 3D model's texture. 


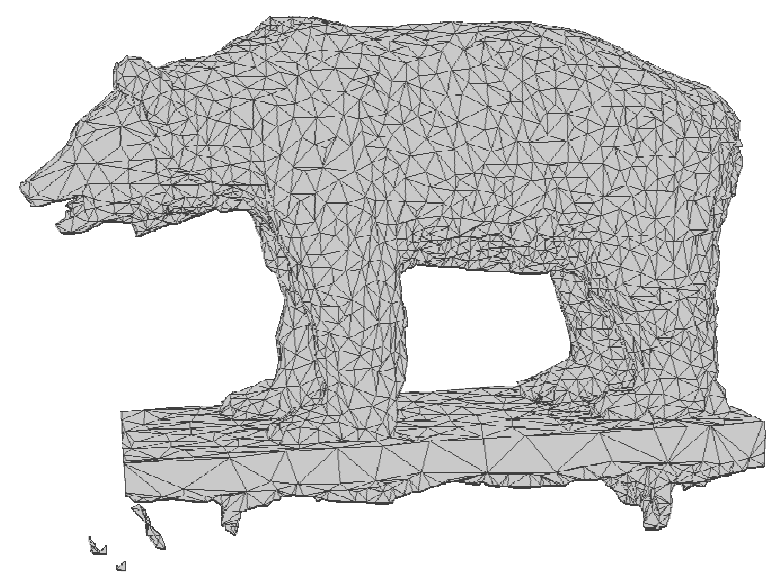

a)



b)

Fig. 5: a) Reconstructed 3D model by photogrammetry, b) Optimized 3D mesh topology.

Finally, we created 6 models of the commonest wild animals in European region. We took into account animals which are causing the highest number of road accidents and which occurrence is also high. Consequently, we created deer, doe, bear, fox, and wolf models. The amount of models can be increased by adding more animals and regions.

\section{Animation}

\subsection{Bones (skeleton)}

In order to animate we have to add a skeleton which will help us during the animation. During the creation of the skeleton we can use presents of animal skeletons, which require only few adjustments for given model. If the skeleton does not meet our requirements, it is possible to create a new skeleton according to needs of the given model. The default bones include control elements for simpler manipulation. They also include control elements of joints, which can be adjusted with the help of shifters in order to change turning force or angle.

\subsection{Bone weight}

In case that the skeleton is assigned to particular parts of the body it is necessary to make so called determination of weights for each bone. It is a determination of force for each bone and how they influence a part of 3D model's mesh points. In other words, it means that if the model's thighbone moves then there is only a movement of the thighbone and no other body part.

\subsection{Walk cycles}

During the walk and run animations we used referential cycles of movement (see Fig. 6) which contain particular phases of movement in particular steps. The amount of these steps is usually 8 or 9 and then the movement is repeated. For more accurate and more natural movement and behaviour it is important to watch the animal on referential video samples. 


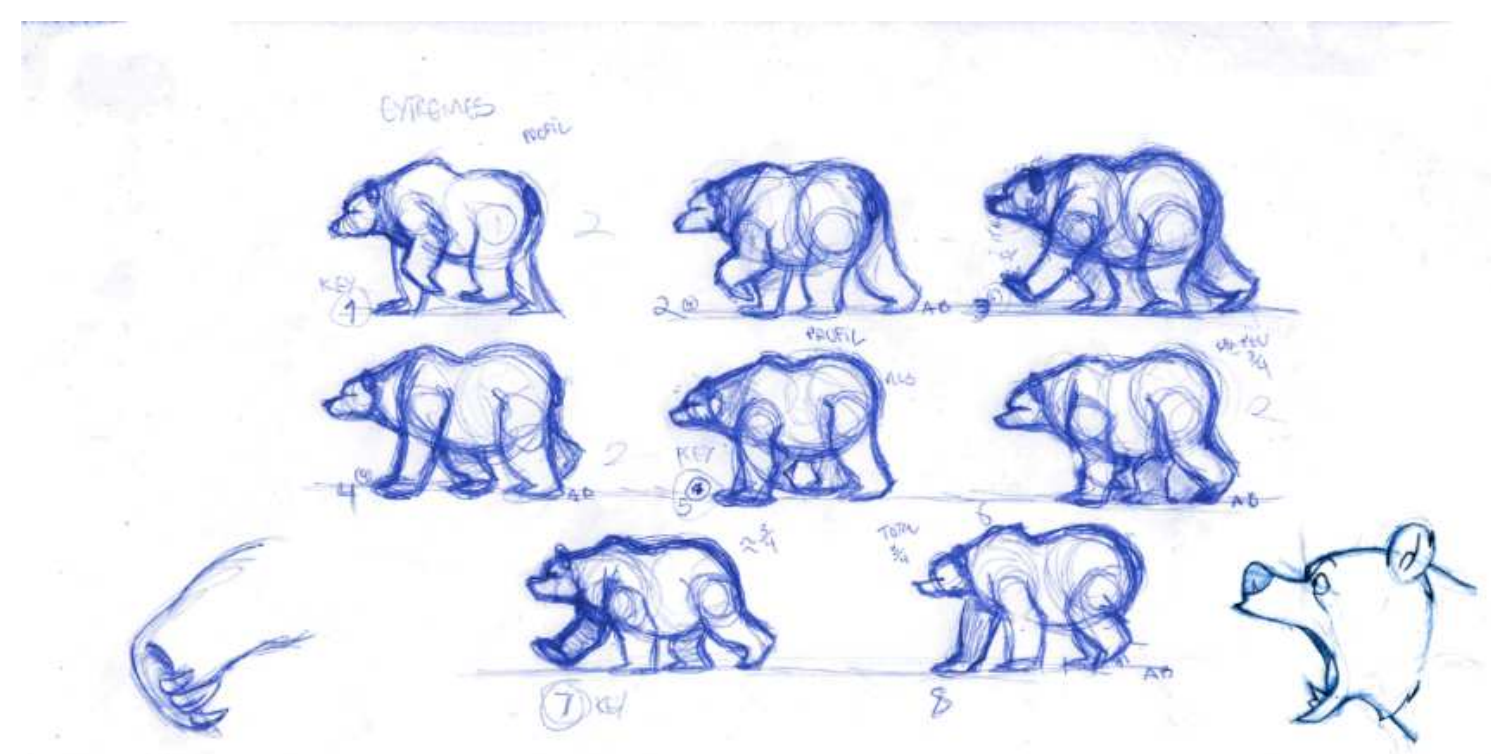

Fig. 6: Bear walk cycles in 8 steps [7].

\subsection{Fur}

In order to make the model look more natural we have to add a fur. After fur addition, it is important to comb the fur in the right direction. Then, we have to adjust undulation, colour, set the thickness and shape according needs and appearance of each animal. An important feature to set is also dynamics. The adjustment of dynamics allows correct undulation and bending changes according to animal's movement. On the Figure $7 \mathrm{~b}$ is shown completed model.

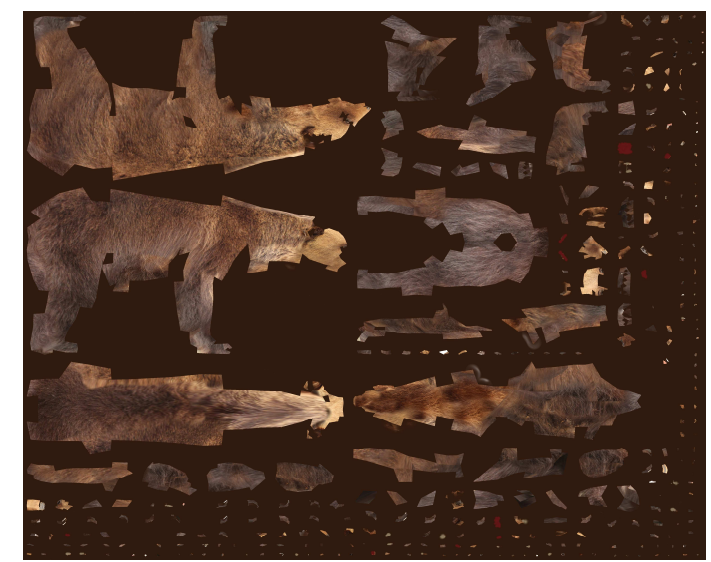

a)

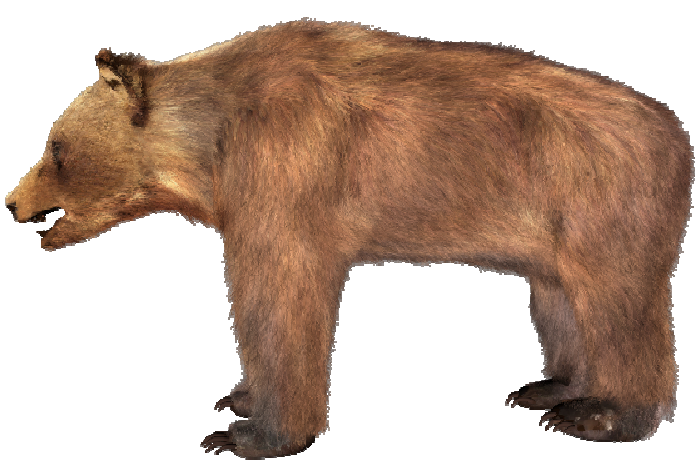

b)

Fig. 7: a) 3D texture of bear, b) Final 3D model with fur.

\section{Scene}

We need to distort the referential video into pictorial sequence. Subsequently, we need to upload the sequence into program, where we can obtain information about the resolution of the image, aspect ratio, focal length, camera chip size, frame rate, and sequence length. All these parameters can be changed according to our needs.

\subsection{Motion tracking}

Motion tracking, also called Match moving, is an essential element when integrating 3D elements with live footage. The idea of motion tracking is well explored and currently utilized for many 
applications. They are further looking for the key points of the 2D image across sequence. These points can be searched separately. Number of points and size of the search field can be changed as needed. After finding the key points, following points are analysed on each frame of the sequence and it is possible to set the minimal length of the images' extent in a certain point of time, error tracking of point in the images and acceleration of a motion of a point in each frame.

\subsection{D reconstruction}

After analysing of all the frames and creating key points, a calculation of $3 \mathrm{D}$ scene can start. If the sequence values are incorrect, during reconstruction it is important to set camera's focal length.

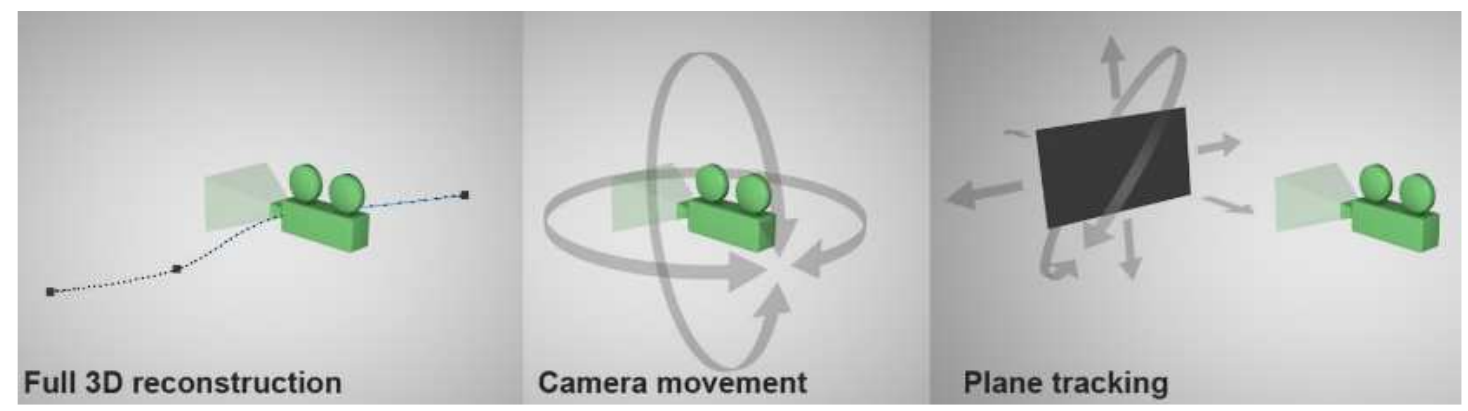

Fig. 8: 3D reconstruction cameras.

The reconstruction of the scene is divided into following stages (see Fig. 8):

- Full Reconstruction: the camera changes its position during the recording and changes a perspective according to recorded objects. This option can easily reconstruct a 3D scene.

- Nodal Pan: the camera is not changing its position; therefore it is impossible to reconstruct points' distance. These points are projected on a spherical area witch a camera in the middle. They are suitable for scenes where camera changes its perspective.

- Plane: it supposes that each track lies on the same level. It is suitable for creation of scenes with green background.



a)

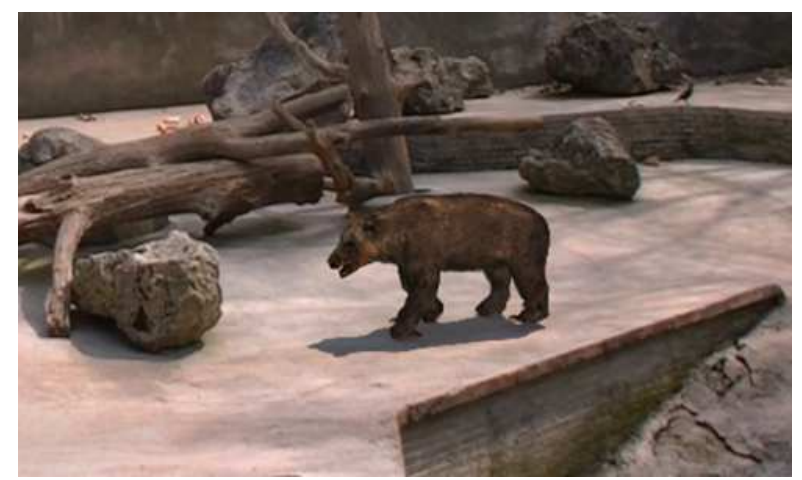

b)

Fig. 9: a) The real scene, b) The virtual 3D model of bear in real scene.

\subsection{Scene configuration}

The animated 3D model of mammals is put into scene (see Fig. 9). In this scene lighting and floor is created. The floor is a crucial element where we can project shadows but the floor will not be visible in the final image render. Lights will be adjusted so the shadow which they create will correspond to shadows in the referential video. We can adjust parameters such as light's source size, light's intensity, colour, sharpness, and intensity of shadow. Later, the scene is completed and we add various filters in order to make 3D model of the animal as reliable as possible in comparison with the referential one. 


\section{Conclusion}

The article describes methods for 3D model creation. In addition, the article involves a detailed description of procedures which were used for 3D model creation and animations which utilized these methods for the most accurate outcome - 3D models of mammals. Furthermore, the article also describes a procedure which was used for a real scene reconstruction where the animated model replaced a living animal. The outcome of our research can be used for algorithm training and testing and after successful testing it can be used as a database for referential videos.

In the near future we would like to undergo a 3D simulation to see another test for different search algorithms. After successful testing, the results are compared with referential videos. In case that comparison brings positive outcome, we would like to focus on other mammals and the development of additional video simulations with various scenarios of mammal migrations. These scenarios will include a random behavior, movement and different mammal species.

\section{Acknowledgement}

The work presented in the paper has been supported by the EUREKA project no. E! 6752 DETECTGAME: R\&D for Integrated Artificial Intelligent System for Detecting the Wildlife Migration.

\section{References}

[1] HELL, P. - PLAVÝ, R. - SLAMEČKA, J. - GAŠPARÍK, J.: Losses of mammals (Mammalia) and birds (Aves) on roads in the Slovak part of the Danube Basin. In: European Journal of Wildlife Research. Volume 51, Issue 1, p. 35 - 40, 2005 - 03. DOI: 10.1007/s10344-004-0068-6.

[2] ALDRIDGE, K. - BOYADJIEV, S.A. - CAPONE, G.T. - DELEON, V.B. - RICHTSMEIER, J.T.: Precision and error of three-dimensional phenotypic measures acquired from 3dMD photogrammetric images. In: Am J Med Genet A. 2005,138 A : 247 - 53. pmid:16158436. DOI: 10.1002/ajmg.a.30959.

[3] WEINBERG, S.M. - NAIDOO, S. - GOVIER, D.P. - MARTIN, R.A. - KANE, A.A. - MARAZITA, M.L.: Anthropometric precision and accuracy of digital three-dimensional photogrammetry: comparing the genex and $3 \mathrm{dmd}$ imaging systems with one another and with direct anthropometry. In: J Craniofac Surg. 2006, 17 : 477 - 483. pmid:16770184. DOI: 10.1097/00001665-20060500000015.

[4] KRIMMEL, M. - KLUBA, S. - DIETZ, K. - REINERT, S.: Assessment of precision and accuracy of digital surface photogrammetry with the dsp 400 system. In: Biomed Tech. 2005, $50: 45$ - 53. DOI: 10.1515/bmt.2005.008.

[5] COOMBES, A. - MOSS, J. - LINNEY, A. - RICHARDS, R. - JAMES, D.: A mathematical method for the comparison of three-dimensional changes in the facial surface. In: Eur J Orthod. 1991, 13 : 95 -110. pmid: 2055258. DOI: 10.1093/ejo/13.2.95.

[6] KAU, CH . - RICHMOND, S. - ZHUROV, A. - KNOX, J. - CHESTNUTT, I. - HARTLES, F. et al.: Reliability of measuring facial morphology with a 3-dimensional laser scanning system. In: Am J Orthod Dentofacial Orthop. 2005 ,128: 424 - 30. pmid:16214622. DOI: 10.1016/j.ajodo.2004. 06.037.

[7] Bear Walking Cycle: http://francorama.blogspot.sk/2013/02/bear-walking-cycle_17.html

[8] Handy laser scanner: http://www.makepartsfast.com/2009/02/685/z-corporations-new-handheld3d-scanners/

[9] YUNSU, BOK - DONG-GEOL, CHOI - IN SO KWEON: Sensor Fusion of Cameras and a Laser for City-Scale 3D Reconstruction. In: Robotics and Computer Vision Lab., Sensors 2014, 14, 20882-20909, p. 20882 - 20909, Korea, ISSN 1424-8220. DOI:10.3390/s141120882. 